\title{
An integrated genomic analysis of gene-function correlation on schizophrenia susceptibility genes
}

\author{
Tearina T Chu and Ying Liu
}

Schizophrenia is a highly complex inheritable disease characterized by numerous genetic susceptibility elements, each contributing a modest increase in risk for the disease. Although numerous linkage or association studies have identified a large set of schizophrenia-associated loci, many are controversial. In addition, only a small portion of these loci overlaps with the large cumulative pool of genes that have shown changes of expression in schizophrenia. Here, we applied a genomic gene-function approach to identify susceptibility loci that show direct effect on gene expression, leading to functional abnormalities in schizophrenia. We carried out an integrated analysis by cross-examination of the literature-based susceptibility loci with the schizophrenia-associated expression gene list obtained from our previous microarray study (Journal of Human Genetics (2009) 54: 665-75) using bioinformatic tools, followed by confirmation of gene expression changes using qPCR. We found nine genes (CHGB, SLC18A2, SLC25A27, ESD, C4A/C4B, TCP1, CHL1 and CTNNA2) demonstrate gene-function correlation involving: synapse and neurotransmission; energy metabolism and defense mechanisms; and molecular chaperone and cytoskeleton. Our findings further support the roles of these genes in genetic influence and functional consequences on the development of schizophrenia. It is interesting to note that four of the nine genes are located on chromosome 6 , suggesting a special chromosomal vulnerability in schizophrenia.

Journal of Human Genetics (2010) 55, 285-292; doi:10.1038/jhg.2010.24; published online 26 March 2010

Keywords: association; bioinformatics; gene expression; schizophrenia; susceptibility; thalamus

\section{INTRODUCTION}

Schizophrenia is one of the most devastating brain diseases of mankind, affecting approximately $1 \%$ of the population worldwide. However, diagnosis of this disease has been based on clinical observations and history with no clear diagnostic tools or biomarkers to date. Recent efforts on advanced molecular psychiatry have characterized schizophrenia as a multi-trait, potentially low-penetrated complexinherited disease covering numerous genetic susceptibility elements, each contributing a modest increase in risk for the disease. Furthermore, the studies of allele-specific single-nucleotide polymorphism (SNP) provide detailed information of disease susceptibility alleles, genotypes or haplotype transmission, regardless major or minor alleles on multiple risk loci on chromosome. ${ }^{1-4}$ Numerous family-based linkage or population-based studies have identified a large set of schizophrenia-associated loci; however, many have not been replicated either in a different ethnic background, ${ }^{5,6}$ or in the same ethnic background but in a diverse geographical location. ${ }^{7,8}$ Although the number of risk loci is rather large, only a small portion overlaps with the large cumulative pool of genes that have shown changes of expression in the disease state. This suggests a heavily weighted portion of the functionally affected genes in schizophrenia that is unlikely to result directly from the susceptibility loci. Instead, these are likely derived from alternative components, for example, epigenetic modification, hormone response and signaling cascades. This is in agreement with the accepted concept that environmental factors have an important role in the pathogenesis of schizophrenia beyond genetic pre-deposition.

The advance of genomewide genetic scanning for susceptibility alleles and genes in schizophrenia, as well as gene expression profiling of affected brain regions in postmortem samples provide great details of the underlying molecular neuropathology of the disease. However, the correlation between inherited risk loci and their functional consequences remains unclear. Nonetheless, change of gene expression is one of the mechanisms that influence biological functions associated with structural variations of DNA or SNPs. Weickert et al. ${ }^{9}$ reported specific genotype or haplotype of SNPs in the estrogen alpha gene is associated with schizophrenia through mechanisms of alternative gene regulation and transcript processing. Nicodemus et al. ${ }^{10}$ found that a minor allele in promoter region of NRG1 increases the risk of schizophrenia, and it correlated with the reduced expression of type III NRG1 isoform. Similarly, Fatemi and colleagues discovered a schizophrenia-associated two-SNP haplotype, located in the intronic 
sequences of PDE4B in the vicinity of a critical splice junction that yields various isoforms. A significant reduction of expression of isoforms 2, 3 and 4 were found in postmortem schizophrenic brain samples. ${ }^{11}$ In addition, based on a pharmacogenomic approach, a susceptibility locus, PDE7B may serve as a predictor for treatment response to risperidone ${ }^{12}$ in patients with schizophrenia. We recently profiled functional transcriptome of neurons from a thalamic subregion, the mediodorsal nucleus, parvocellular division (MDNp) that comprises a thalamo-cortical circuit involved in working memory, a deficit in persons suffering from schizophrenia. Despite the identification of a large set of genes showing changes of expression in this thalamic subregion, few served as primary genetic contributors previously implicated in the disease, thus presenting a direct gene functional effect on pathological consequences. Therefore, our aim was to genomically identify dysregulated genes from the thalamic gene expression findings, whose loci have also been reported as significantly associated with schizophrenia from various analyses of association studies using a bioinformatic approach. The change of expression of these thalamic-specific candidate genes was subsequently confirmed by qPCR.

\section{MATERIALS AND METHODS}

\section{Study cohorts, RNA preparation and amplification}

The same set of total RNAs of neurons from the mediodorsal nucleus, parvocellular (MDNp) subregion of the thalamus prepared for the previous microarray assay was used in this study. ${ }^{13}$ Briefly, frozen postmortem tissue (Neuropathology Consortium) was donated by the Stanley Medical Research Institute (Chevy Chase, MD, USA) brain collection ( $n=15 \mathrm{SZ}, n=15 \mathrm{NC}$ ). The delineation, laser capture dissection of neurons and RNA isolation were described previously. ${ }^{13}$ RNA quality was assessed again prior to use by the Agilent Bioanalyzer pico RNA assay (Agilent Technologies, Palo Alto, CA, USA). Those which showed two peaks of $18 \mathrm{~S}$ and $28 \mathrm{~S}$ ribosomal RNA (rRNA), regardless the levels of degradation, were subjected to amplification (data not shown). In all, 5-10 ng of total RNA were reverse transcribed and amplified using WT Ovation PCR amplification kit (Nugen, San Carlos, CA, USA). The resulting single-stranded cDNA for each subject was assessed by the Bioanalyzer pico RNA assay. The length of the amplified cDNA ranged between 200 and 2000 nucleotides, with peaks of 300-600 nucleotides. cDNA showing significant shift (to the left) on size distribution was removed from further analysis.

\section{Preparation of SZ-associated functional gene list with altered gene expression}

To compile the functional candidate gene list, we initially adopted the previous gene list derived from two-way analysis of variance (ANOVA, disease-pH) of genomewide profiling of 23 postmortem brains ( $11 \mathrm{SZ}$ vs $12 \mathrm{NC}$ ).${ }^{13}$ In order to reduce false negative selection and be more inclusive for the subsequent expression guided genetic candidacy screening, we employed an alternative statistical algorithm by carrying out a Welch test on the same microarray data set, so to improve statistical power by an increase of degree of freedom. To control the potential increase of the false-positive selection, the list was further corrected by Benjamini and Hochberg multi-testing algorithm, ${ }^{14}$ and genes were selected with corrected $P<0.05$, followed by a removal of diseaseirrelevant, pH-dependent genes obtained from meta-analysis of 43 brains across three psychiatric diseases and normal control cohorts. ${ }^{13}$ The chip inclusion and filter criteria of signal intensity $(S \geqslant 16)$ and fold-change $(\mathrm{FC} \geqslant 1.5)$ for gene selection were used the same as in the two-way ANOVA. The combined list was used for further analysis.

\section{Preparation of SZ susceptibility gene list, chromosomal mapping and correlation analysis}

We downloaded the 'SchizophreniaGene' database (updated 23 September 2009), containing multiple fields of chromosome residency, gene symbol, NCBI Entrez Gene ID, encoded protein, study and polymorphism for each of the SZ susceptibility gene from the 'Schizophrenia Research Forum', a public website (http://www.schizophreniaforum.org/) that provides a systematic collection of genetic association studies carried out on schizophrenia phenotypes published on peer-reviewed journals. ${ }^{2}$ To this gene list of 786 genes, we then added 2 genes identified by runs of homozygosity in schizophrenia, ${ }^{15}$ and 21 genes revealed by structural breakage of microdeletions and duplications ${ }^{16}$ to the SZ susceptibility list. This compilation produced a list of 809 of genes, which we used for our further analyses (data not shown). The macroscopic view of SZ gene distribution mapped on chromosomes was generated by ArrayAssist (Stratagene, now Agilent Technologies). The chromosomal enrichment of SZ genes was analyzed by a modified Fisher's exact test. The probability (onetailed/right) of SZ genes falling on each chromosome in comparison to the background gene-chromosome distribution was calculated by penalizing (subtracting) the count of positive agreement by 1. Functional analysis and gene annotation were based on Gene Ontology, integrated annotation databases within DAVID ${ }^{17,18}$ and Genecards (http: Iwww.genecards.org), as well as features in Genespring (Agilent Technologies). To correlate the expression and susceptibility gene list, a relational database primarily keyed by 'gene symbol' was created in Microsoft Access. For each of the overlapping genes, original study publications (listed in the downloaded SchizophreniaGene database) were reviewed for association accuracy and for individual SNP results. The intragenic location of each positive SNP and its functional effect of gene products were also examined and confirmed with NCBI SNP database.

\section{Real-time PCR and data analysis}

A total of $2 \mathrm{ng}$ of amplified cDNA were used in each real-time PCR assay. All assays were carried out in triplicate using SyBR-Green on ABI 7900HT realtime PCR machine. The primer pair sequences were designed using Primer 3 plus software (http://www.bioinformatics.nl) with input sequence within $1 \mathrm{~kb}$ from 3' end of the mRNA, yielding an amplicon between 100 and $200 \mathrm{bp}$. All primer pairs were validated by endpoint PCR and gel electrophoresis before real-time PCR assay. Three reference genes, ribosomal protein S11 (RPS11), chromosome 9 open reading frame 6 (C9orf6) and chromosome 1 open reading frame 122 (Clorf122) showing minimum expression fluctuation among all samples were used in each set of assay. The calculation and normalization of $\mathrm{Ct}$ value and $\Delta \mathrm{Ct}$ value for each gene and for each subject were carried out as described previously. ${ }^{13} \mathrm{~A}$ Welch test (unpaired, one-tailed and unequal variance) was used to determine the significance of the target gene at a cut-off of $P<0.05$ (uncorrected) in Excel. Bonferroni's correction for multiple qPCR gene confirmation was not carried out as our analysis, which was based on a priori hypothesis of positive microarray results of the target genes.

Owing to the profound effect of low brain $\mathrm{pH}$ on RNA and profiling data quality observed from our previous genomewide microarray analysis, ${ }^{13}$ data points from brains with $\mathrm{pH}<6.0$ were excluded from the analysis. In addition, $\Delta \mathrm{Ct}$ data points outside the three s.d. from the mean, covering $99 \%$ of all data point population were also excluded from the statistical calculation to control the random effect of real-time PCR failure. The mean fold change of gene expression was calculated from $\Delta \Delta \mathrm{Ct}(\mathrm{SZ} \Delta \mathrm{Ct}-\mathrm{NC} \Delta \mathrm{Ct})$.

\section{RESULTS}

In all, 1141 genes passed the significance analysis by Welch test, with multi-testing and $\mathrm{pH}$ corrections (data not shown). Among these, $78 \%$ of genes overlapped with the previous two-way ANOVA gene list with additional 499 selected genes. The combined list containing a total of 1680 differentially expressed genes were subject to screening for functional influenced schizophrenia susceptibility genes.

A total of 809 Schizophrenia genes were cumulated either from Schizophrenia Research Forum website on 23 September 2009 update or manually curated from literature. When mapping these genes on genome, they spread on all chromosomes with a few visually dense 'hot spots' on chromosome 6, 8, 10, 19 and 22 (Figure 1). In order to determine whether a chromosome harbors higher vulnerability, a modified Fisher's exact test was carried out on affected loci of each chromosome in relevance to all loci resident on the chromosome. The result showed chromosome 5, 6, 10 and 22 were significantly more vulnerable $(P<0.05)$ in susceptibility to schizophrenia, with highly 


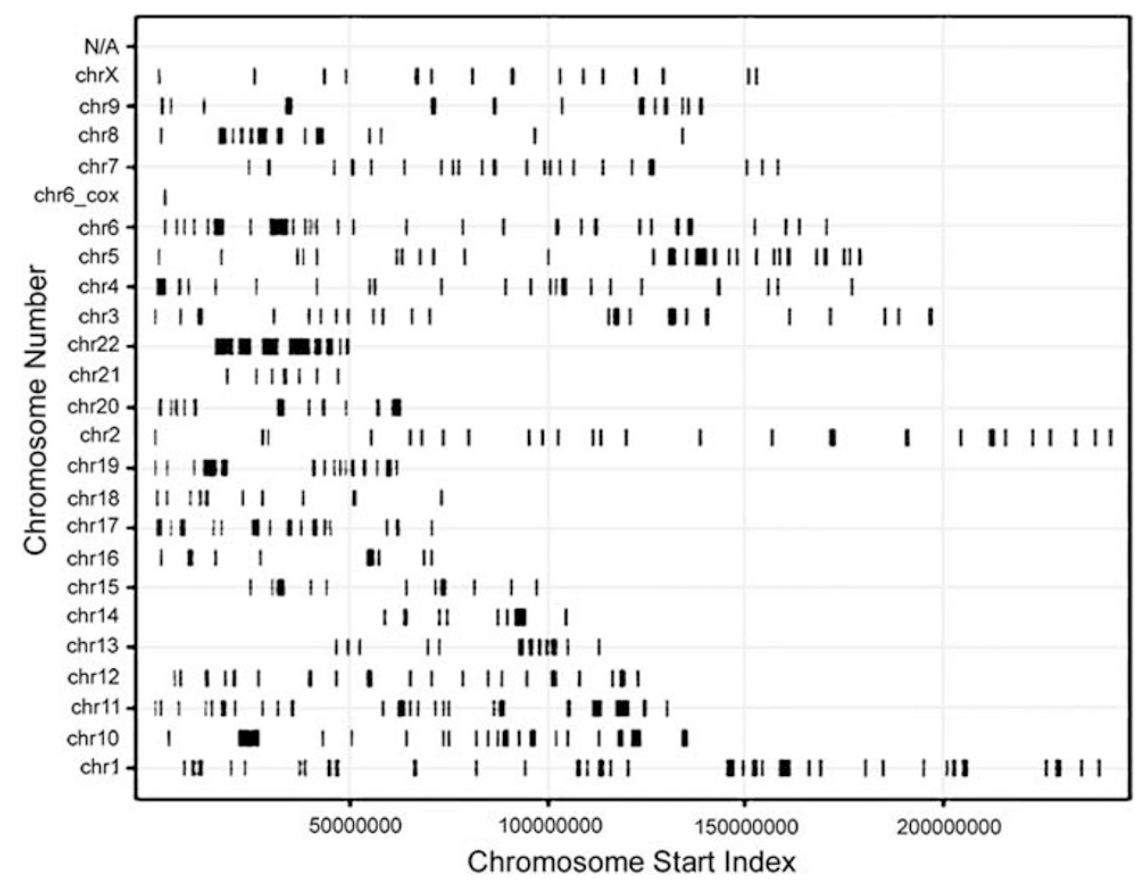

Figure 1 Genome mapping of schizophrenia susceptibility loci. Schizophrenia susceptibility genes were mapped on chromosomes as vertical lines according to the Chromosome Start Index or blocks spanning the corresponding chromosomal regions.

Table 1 Schizophrenia-susceptibility-associated chromosomal vulnerability

\begin{tabular}{lccl}
\hline Chromosome no. & Target hits & Publication hits & Positive P-value \\
\hline Chr1 & 66 & 2154 & 0.966567 \\
Chr2 & 34 & 1366 & 0.997402 \\
Chr3 & 36 & 1133 & 0.882021 \\
Chr4 & 26 & 776 & 0.78363 \\
Chr5 & 51 & 904 & 0.002841 \\
Chr6 & 81 & 1101 & $4.58 \mathrm{E}-09$ \\
Chr7 & 23 & 1009 & 0.997965 \\
Chr8 & 29 & 706 & 0.37104 \\
Chr9 & 21 & 802 & 0.977797 \\
Chr10 & 48 & 823 & 0.002029 \\
Chr11 & 43 & 1191 & 0.639968 \\
Chr12 & 34 & 1084 & 0.893615 \\
Chr13 & 17 & 389 & 0.361842 \\
Chr14 & 15 & 681 & 0.995082 \\
Chr15 & 17 & 653 & 0.970757 \\
Chr16 & 12 & 884 & 0.999998 \\
Chr17 & 28 & 1168 & 0.997486 \\
Chr18 & 317 & 0.623186 \\
Chr19 & 12 & 1358 & 0.99827 \\
Chr20 & 33 & 595 & 0.695461 \\
Chr21 & 21 & 234 & 0.361559 \\
Chr22 & 11 & $\mathbf{5 1 9}$ & $\mathbf{8 . 9 4 E - 2 9}$ \\
Chrx & $\mathbf{8 2}$ & 781 & 0.981685 \\
\hline
\end{tabular}

The positive correlation of probability was calculated by a modified Fisher's exact test on a total of 760 schizophrenia risk hits against a total of 20628 population hits. Bold: significant $(P<0.05)$ positive correlation.

significant targets on chromosomes $6(P=4.58 \mathrm{E}-09)$ and 22 $(P=8.94 \mathrm{E}-29)$ (Table 1). Functional annotation of schizophrenia genes using Gene Ontology revealed very significant $P$ values for categories involving transmission of nerve impulse, synaptic transmission, cell surface receptor-linked signal transduction, neurite differentiation and migration, cell death and neuro-development (Supplementary Table 1). It is worthy to note that there was some degree of functional bias on the tested candidate genes for susceptibility that derives from theoretical approaches to schizophrenia or expression literature rather than a random selection in many association studies.

As expected, among 1680 differentially expressed genes, only 57 genes $(3.4 \%)$ overlapped with the cumulated 809 SZ susceptibility genes. This number was further reduced to 25 genes (1.5\%) for those that have shown a positive result for at least one SNP in at least one association study after the manual review of the original publications for each of the 57 genes (supplementary Table 2). This reduction indicates the necessity for a manual confirmation of association results when compiling evidence-based SZ-associated susceptibility gene candidates.

We carried out real-time PCR assays on 19 SZ genes in 21 brains ( $10 \mathrm{SZ}$ vs $11 \mathrm{NC}$ ) to validate the gene expression changes. The results are summarized in Table 2. Nine susceptibility genes, $C H G B$, SLC18A2, SLC25A27, ESD, C4A/C4B, TCP1, CHL1 and CTNNA2 were shown to have significant expression changes in schizophrenic postmortem brain samples. It is interesting to note that their associated SNPs were mostly located in the non-coding region of intron or untranslated regions of the gene except for CHL1 and CHGB, suggesting an indirect mechanism in effect for their functional influence of gene expression from the allele-specific susceptibility loci. In CHL1, a SZ-associated SNP (rs2272522) was found in exon 3 involving a Leu $\rightarrow$ Phe change of the protein, ${ }^{19,20}$ whereas in $C H G B$, three SNPs (rs236152, rs236155 and rs6133278) were found, involving mis-sense changes of the protein, Ala $\rightarrow$ Gly, Arg $\rightarrow$ Gln and Asp $\rightarrow$ Asn, respectively ${ }^{21,22}$ (Table 3 ). These susceptibility genes are involved in multiple critical cellular functions, which may be relevant to the 
Table 2 Summary of schizophrenia-susceptibility associated gene expression changes by qPCR

\begin{tabular}{lllc}
\hline Gene symbol & Genbank ID & Description & Pold change \\
\hline CTNNA2 & NM_004389 & Catenin (cadherin-associated protein), alpha 2 & 0.003042 \\
CHL1 & NM_006614 & Cell adhesion molecule with homology to L1CAM (close homolog of L1) & 0.036009 \\
C4A/C4B & NM_007293/ NM_001002029 & Complement component 4A/4B & 1.69447 \\
SLC25A27 & NM_004277 & Solute carrier family 25, member 27 & 0.022013 \\
TCP1 & NM_001008897 & t-complex 1 & 3.368363 \\
SLC18A2 & NM_003054 & Solute carrier family 18 (vesicular monoamine), member 2 & 0.005634 \\
ESD & NM_001984 & Esterase D/formylglutathione hydrolase & -2.59986 \\
CHGB & NM_001819 & Chromogranin B (secretogranin 1) & 0.004782 \\
\hline P & -1.84754 \\
\hline
\end{tabular}

$P$-value (uncorrected): Welch test against 21 postmortem brain samples (10 SZ vs $11 \mathrm{NC}$ ). Fold change: mean fold change of SZ to NC.

molecular underpinnings involved in schizophrenia and are discussed individually below.

\section{DISCUSSION}

Change of gene expression is one of the mechanisms that influence biological functions associated with structural variations of DNA or SNPs in disease. ${ }^{23}$ Here we reported qPCR validated functionally associated schizophrenia susceptibility genes identified in a whole human genome screening of individually harvested and accumulatively collected neurons from a thalamic subregion (MDNp) which is reciprocally connected to dorsolateral prefromal cortex, a cortical region involved in working memory.

For nearly 50 years, researchers have attempted to map genes for schizophrenia; however, these efforts have resulted in inconclusive findings primarily because of the problems of linkage in complex traits diseases, such as schizophrenia, and the failure to replicate positive results across different genetic studies. The underlying genetic issues may involve an unclear mode of transmission and locus heterogeneity. In addition, it is hard to definitively define affected and unaffected family members in multiple affected pedigrees that often contain mild cases or relatives with spectrum disorders for family-based studies. Thus, we excluded the candidate genes that were solely suggested by family-based linkage studies from this study. Nonetheless, there have been reports of linkage between schizophrenia and genetic markers on 14 different chromosomes (1, 2, 4, 5, 6, 7, 8, 9, 10, 13, 15, 18, 22 and X), covering thousands of genes. ${ }^{24}$ Although, it is difficult to separate true positive findings from noise, the strongest evidence pointed to involvement of chromosomes $8 p, 13 q$ and $22 q$ from meta-analysis of genome linkage studies. ${ }^{25,26}$ These findings are in agreement with our genomic mapping of schizophrenia susceptibility loci (Figure 1), especially chromosome 22 being one of the most vulnerable chromosomes based on the integrated results of literature-based association studies (Table 1).

As a major gift from decoding the human genome, a detailed map containing millions of SNPs on every chromosome was created, ${ }^{27}$ which made possible the study of genomewide association on complex traits diseases. The aim of the association is to detect a significant relationship between the disease and a particular allele or haplotype in unrelated cases and controls. These efforts have narrowed the schizophrenia susceptibility loci down to a few hundred genes. However, they are still widely distributed on almost every chromosome (Figure 1). Similar to linkage studies, there have been difficulties in the replication of positive findings in various association studies. One of the reasons may be involved in the mixture or diversity of ethnic groups that differ in the frequency of the disease. In addition, most association studies conducted so far either focus on the polymorph- isms within or near a gene, which is a 'functional' candidate of schizophrenia, or within DNA regions that were previously identified by linkage studies. Thus, the present schizophrenia susceptibility gene-chromosome distribution map (Figure 1) does not present genes with an 'equal opportunity', but rather reflects the preferential selection of genes on functional genetic basis. Nonetheless, owing to the recent break-through of the development of high-density SNP arrays, several large population-based association studies provide unbiased genome scans in search of schizophrenia susceptibility genes. ${ }^{1,28}$ Various statistically significant markers spanning a region of HMC loci and histone gene cluster on chromosome 6p21.3-22.1, a marker located upstream of the neurograinin gene $(N R G N)$, a marker in intron 4 of transcription factor $4,{ }^{1}$ and markers in genes including CACNA1C, CSF2RB, CACNA1B and DGKI have been identified. $^{28}$ These findings are partially consistent with our immune-related findings using expression-genetic approach.

Our results support the general observations that only a small portion of schizophrenia susceptibility loci overlaps with the large cumulative pool of genes showing changes of expression in disease state. The possible reasons include (1) many of the risk genes are false positive findings; (2) the effect of susceptibility genes goes beyond its own expression, and toward 'downstream' or 'spider-web' connectivity; and (3) the function of susceptibility alleles to schizophrenia is through alternative mechanisms instead of direct change of gene expression. Despite some risk genes being found with altered expression levels in postmortem schizophrenic brains, one common alternative mechanism linking SNP-gene expression-disease is that a specific allele, often residing in an intron or the promoter region, is associated with generation of unique spliced variants and/or change of expression ratio among variants of the gene. Recent studies on DISC1, $B A B R B 2, D R D 2$ and $A K T 1$, top-ranked SZ susceptibility genes based on meta-analysis of multiple case-control association studies all support this allele-expression regulatory mechanism. ${ }^{29-33}$ However, the search for connection between risk genetic polymorphisms and brain GRIN2B mRNA levels, another top-ranked risk gene in SZ, resulted in negative findings. As such, the study focused on the overall mRNA level, not on variants. ${ }^{34,35}$

In total, $57 \mathrm{SZ}$ risk genes overlapped with our differential expression gene list findings from whole genome screening of thalamic neurons in postmortem schizophrenic brains. Among these, only 25 genes were reported for at least one positive SNP finding in all association studies (Supplementary Table 2). Hence, a manual review and correction was shown to be necessary when carrying out an integrated analysis of genetic and expression data using literature-based bioinformatic tools. We tested 19 genes, and 9 were qPCR confirmed with significant differential gene expression in schizophrenic postmortem brain 


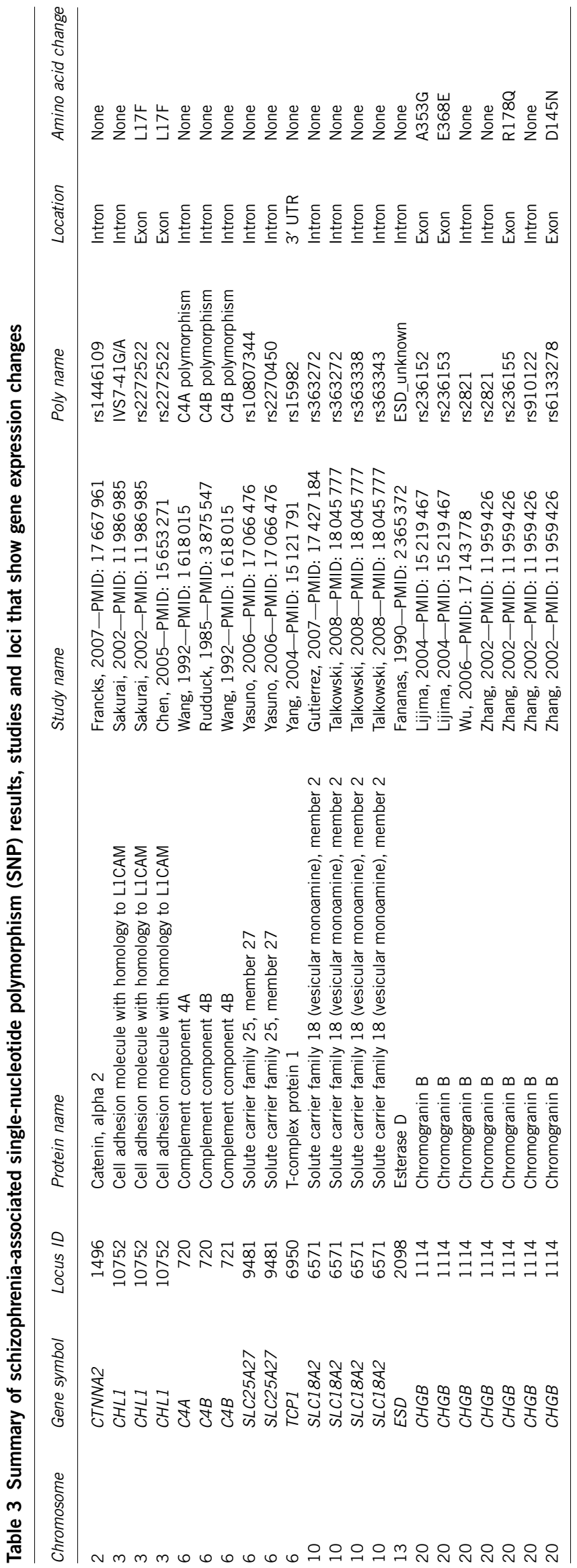

samples vs controls (Table 2). Among the negative qPCR findings, we cannot exclude the possibility that the SNP-expression correlations occur at the variant's level. Further investigation of associated mechanisms is necessary. In addition, top-ranked SZ genes, such as GABRB2, DAOA and DRD2, did not appear on our final SZ candidate list. This may be because of the specific expression pattern of the thalamic neuronal cells used in our expression analysis. The nine qPCR confirmed genes, CHGB, SLC18A2, SLC25A27, ESD, C4A/C4B, TCP1, CHL1 and CTNNA2, will be individually discussed in functional context below.

\section{Synapse and neurotransmission}

An association between chromogranin B (CHGB) polymorphisms and SZ was reported in both Chinese Han and Japanese populations, in which linkage disequilibrium was found in multiple pairs of SNPs in exon 4, resulting in amino acid changes of the coded protein. ${ }^{21,22}$ The CHGB gene encodes for chromogranin $\mathrm{B}$, which belongs to a family of soluble proteins stored and released from large dense-core secretory vesicles at the synapse. ${ }^{36,37}$ These granins have an important role in the sorting and aggregation of secretory products in the transGolgi network and in the subsequent formation of secretory granules. ${ }^{38}$ A reduction of chromogranin B levels in cerebrospinal fluid (CSF), as well as chromogranin B-like immunoreactivity in distinct subregions of the hippocampus were reported in patients with SZ. ${ }^{39,40}$ Its biological function as potential precursor of active neuropeptide and disease-associated mechanism(s) remain uncharacterized. ${ }^{41}$ Given that chromogranins $\mathrm{A}$ and $\mathrm{B}$ are present in human cerebrospinal fluid at higher concentration than in plasma, and have a role in the biochemistry of the synapses, they may serve as markers for large dense-core synaptic vesicle function and pathology. ${ }^{39,42}$ Potential drug effects for the microarray screening of candidate genes were generally discussed previously. ${ }^{13}$ Although Landén et al. ${ }^{39}$ reported no correlation between the levels of the chromogranins and lifetime or recent neuroleptic dose, as well as medicated or drug-free status in SZ, contradictory results were reported in other studies. ${ }^{43-45}$ The expression of chromogranin A may be significantly influenced by neuroleptic medication, as a reduction of cerebrospinal fluid chromogranin A level was demonstrated in patients with SZ chronically treated with neuroleptic medications. ${ }^{44}$ Nonetheless, the correlation between SZ susceptibility, CHGB expression and possible drug effect requires further investigation. In addition, allelic SNPs have been implicated in the regulation of gene expression. ${ }^{29}$ Further studies on the polymorphic variants of CHGB and their corresponding levels of expression, as well as the functional influences due to the change of amino acid of the protein are needed to further the understanding of the potential disease-associated mechanisms of CHGB in schizophrenia.

The SLC18A2 gene codes for a vesicular transporter that pumps cytosolic monoamines, including dopamine, norepinephrine, serotonin and histamine, into synaptic vesicles in an ATP-dependent manner, which facilitate biogenic amine neurotransmission. Altered storage, release and reuptake of dopamine may contribute to both the hypoand hyperdopaminergic states that exist in various diseases, including schizophrenia. A recent computational model reveals that the dopamine transporter (DAT), the vesicular monoamine transporter (VMAT2) and the enzyme monoamine oxidase (MAO) are the most influential components controlling the synaptic level of dopamine and the formation of toxic intracellular metabolites, which lead to the abnormality of brain functions in diseases. ${ }^{46}$ Significantly elevated platelet vesicular monoamine transporter density through high-affinity binding assay was observed in the mixed population of SZ patients, suggesting a potential role as biomarker. ${ }^{47}$ Two risk haplo- 
types of SLC18A2 were reported for both SZ and bipolar mood disorder in a case-control study of Spanish population. ${ }^{48}$ This leads to support an arguable concept that these two psychiatric disorders share common genetic determinants, ${ }^{49}$ which is strongly supported by a recent large population study in Swedish families. ${ }^{50}$

\section{Energy metabolism and defense mechanisms}

The gene product of SLC25A27, UCP4 is a member of mitochondrial uncoupling proteins that create proton leaks across the inner mitochrondrial membranes, thus uncoupling oxidative phosphorylation from ATP synthesis in a form of exchange of energy with heat. This dysregulation of energy production may be involved in abnormal metabolism in the brain, which is often observed in schizophrenic patients. ${ }^{51,52}$ The SLC25A27 transcripts were brain specific and detected in various brain tissues. ${ }^{53}$ UCP4 has demonstrated neuronal protection by mediating an adaptive shift in energy metabolism in response to metabolic and oxidative stress, potentially by the regulation of mitochondrial $\mathrm{Ca}^{2+}$ signaling. ${ }^{51,54}$ It is interesting to note that another neuronal protective SZ gene, ESD codes for an esterase, involved in the formation of glutathione, an antioxidant molecule by detoxification of formaldehyde. ${ }^{55}$ This enzyme may also be associated with the recycling of sialic acids, involved in the epitopes of gangliosides recognized by the immune system in the central nervous system. ${ }^{56}$ In addition, ESD gene has been used as a genetic marker for retinoblastoma and Wilson's disease. ${ }^{57}$ The latter has demonstrated one of the two predominant psychiatric manifestations, schizophreniaform-illness, suggesting an additional correlation of ESD and SZ ${ }^{58}$

Our other immune-related findings, $C 4 A / C 4 B$ gene loci localize to the major histocompatibility complex (MHC) class III region on chromosome 6. The $C 4 A / C 4 B$ gene encodes the basic form of complement factor 4 , involved in the classical activation pathway of the complement system, a major effector of the innate and adaptive immune systems. Compromised complement activity is associated with vulnerability to infection, apoptotic cell clearance and autoimmunity. The $\mathrm{C} 4 \mathrm{~A}$ and $\mathrm{C} 4 \mathrm{~B}$ proteins are isoforms of $\mathrm{C} 4$, which is expressed as a single-chain precursor undergoing proteolytic cleavage on activation. The proteolytic derivative of $\mathrm{C} 4 \mathrm{a}$, anaphylatoxin is a mediator of local inflammatory process. ${ }^{59}$ The $\mathrm{C} 4 \mathrm{~b}$ is the major activation product in the classical complement pathway. $\mathrm{C} 4 \mathrm{~A}$ and $\mathrm{C} 4 \mathrm{~B}$ are highly polymorphic with variation at many residues, but the difference between their protein activities correlates with differences in only four amino acids. $^{60,61}$ It is therefore difficult to measure their concentration separately. ${ }^{62}$ A significant increase in the frequency of homozygous C4B deficiency was found among Swedish patients with SZ compared with controls, ${ }^{63}$ although others reported contradictory results. ${ }^{64,65}$ Nonetheless, C4B serum protein level has been found to be significantly decreased in the SZ patients. ${ }^{64}$ The detailed mapping of the expression of $\mathrm{C} 4 \mathrm{~A}$ or $\mathrm{C} 4 \mathrm{~B}$ gene that is associated with $\mathrm{SZ}$ requires further investigation. Recent meta-analyses of combined SNP data from several large genomewide scans suggests a significant association with several markers spanning the HMC region on chromosome $6 .^{1,28}$ This finding and our gene-expression results lead further support to an immune component being involved with schizophrenia risk and functionality.

\section{Molecular chaperone and cytoskeleton}

TCP1 encodes a molecular chaperone which assists the proper folding of proteins, including actin, in an ATP-dependent manner. ${ }^{66}$ Although this gene has not been widely studied in SZ except for its genetic association to the disease, both protein folding and protein degradation have been shown to be the most prominent biochemical deficits observed in schizophrenia. ${ }^{13}$ Actin, an important substrate of TCP1, forms a framework for the dendritic spines, on which most excitatory nerve impulses are transmitted. It has been reported that the prefrontal cortex of subjects with SZ contains fewer dendritic spines. ${ }^{67}$ Improper folding of actin or other functional related molecules, indirectly influenced by TCP1, may contribute to the cytoskeleton deficit in multiple brain areas in SZ postmortem samples.

CHL1 codes for a protein member of the $L 1$ gene family of neural cell adhesion molecules that regulate cell migration during nervous system development and regeneration. CHL1 has been shown to promote neurite outgrowth of cerebellar and hippocampal neurons, and suppress neuronal cell death. ${ }^{68,69}$ In human, deletion or alteration of the CHL1 gene may contribute to mental defects associated with the $3 \mathrm{p}$-syndrome, caused by distal deletions of the short ( $\mathrm{p}$ ) arm of chromosome 3, and schizophrenia. ${ }^{20,70,71}$ CHL1-deficient mice have been shown to have aberrant connectivity of hippocampal mossy fibers and olfactory sensory axons, which resulted in behavior change secondary to the disruption of information processing. ${ }^{72}$ Consistently, disorganization and sensorimotor-gating impairment abnormalities have been demonstrated in multiple brain regions, (for example, thalamus) in SZ brains. ${ }^{73}$

Alpha-catenins are also cytoskeletal proteins, encoded by three CTNNA genes, in which CTNNA2 is primarily expressed across the central nervous system. ${ }^{74}$ Similar to CHL1, CTNNA2 also plays a role in regulating hippocampal development and sensorimotor gating. ${ }^{75}$ Its expression is critical for maintaining the stability of dendritic spines in rodent hippocampal neurons in culture. ${ }^{76}$ These multiple layers of defects in brain cytoskeleton structure caused by three susceptibility genes may induce magnified functional deficits in schizophrenia.

Our qPCR findings of nine expression-guided SZ susceptibility genes are involved in multiple key biological and disease-associated processes in the central nervous system. These genes or gene products demonstrate a high-degree of functional connectivity, thus potentially leading to confounding neuropathological outcomes of $\mathrm{SZ}$ in a convergent manner. It is interesting to note that four of the nine genes identified $(C 4 A / C 4 B, T C P 1$ and SLC25A27) localizes to chromosome 6 (spanning p21.3, q25.3 and p11.2), including two $(\mathrm{C} 4 \mathrm{~A} / \mathrm{C} 4 \mathrm{~B})$ in the highlighted vulnerable regions of major histocompatibility complex. These findings further support immune involvement and the special chromosomal association of chromosome 6 to schizophrenia.

\section{ACKNOWLEDGEMENTS}

We are grateful for the support of the Microarray Shared Research Facility at Mount Sinai School of Medicine. This work was supported by The Stanley Medical Research Institute grant.

1 Stefansson, H., Ophoff, R. A., Steinberg, S., Andreassen, O. A., Cichon, S., Rujescu, D. et al. Common variants conferring risk of schizophrenia. Nature 460, 744-747 (2009).

2 Bertram, L. Genetic research in schizophrenia: new tools and future perspectives. Schizophr. Bull. 34, 806-812 (2008).

3 Miller, C. L., Murakami, P., Ruczinski, I., Ross, R. G., Sinkus, M., Sullivan, B. et al. Two complex genotypes relevant to the kynurenine pathway and melanotropin function show association with schizophrenia and bipolar disorder. Schizophr. Res. 113, 259-267 (2009).

4 Stefanis, N., Trikalinos, T., Avramopoulos, D., Smyrnis, N., Evdokimidis, I., Ntzani, E. et al. Association of RGS4 variants with schizotypy and cognitive endophenotypes at the population level. Behav. Brain Funct. 4, 46 (2008).

5 Wei, J. \& Hemmings, G. P. Lack of a genetic association between the frizzled-3 gene and schizophrenia in a British population. Neurosci. Lett. 366, 336-338 (2004) 
6 Zhang, Y., Yu, X., Yuan, Y., Ling, Y., Ruan, Y., Si, T. et al. Positive association of the human frizzled 3 (FZD3) gene haplotype with schizophrenia in Chinese Han population. Am. J. Med. Genet. B Neuropsychiatr. Genet. 129B, 16-19 (2004).

7 Liu, Y. L., Fann, C. S. J., Liu, C. M., Chang, C. C., Yang, W. C., Hung, S. I et al. More evidence supports the association of PPP3CC with schizophrenia. Mol. Psychiatry 12, 966-974 (2007).

8 Xi, Z., Yu, L., Shi, Y., Wei, Q., Zheng, Y., Zhang, J. et al. No association between PPP3CC and schizophrenia in the Chinese population. Schizophr. Res. 90, 357-359 (2007).

9 Weickert, C. S., Miranda-Angulo, A. L., Wong, J., Perlman, W. R., Ward, S. E., Radhakrishna, V. et al. Variants in the estrogen receptor alpha gene and its mRNA contribute to risk for schizophrenia. Hum. Mol. Genet. 17, 2293-2309 (2008).

10 Nicodemus, K. K., Law, A. J., Luna, A., Vakkalanka, R., Straub, R. E., Kleinman, J. E et al. A 5' promoter region SNP in NRG1 is associated with schizophrenia risk and type III isoform expression. Mol. Psychiatry 14, 741-743.

11 Fatemi, S. H., King, D. P., Reutiman, T. J., Folsom, T. D., Laurence, J. A., Lee, S. et al. PDE4B polymorphisms and decreased PDE4B expression are associated with schizophrenia. Schizophr. Res. 101, 36-49 (2008).

12 Ikeda, M., Tomita, Y., Mouri, A., Koga, M., Okochi, T., Yoshimura, R. et al. Identification of novel candidate genes for treatment response to risperidone and susceptibility for schizophrenia: integrated analysis among pharmacogenomics, mouse expression, and genetic case-control association approaches. Biol. Psychiatry 67, 263-269 (2010).

$13 \mathrm{Chu}$, T. T., Liu, Y. \& Kemether, E. Thalamic transcriptome screening in three psychiatric states. J. Hum. Genet. 54, 665-675 (2009).

14 Benjamini, Y. \& Hochberg, Y. Controlling the false discovery rate: A practical and powerful approach to multiple testing. J. Roy. Statist. Soc. Ser. B 57, 289-300 (1995).

15 Lencz, T., Lambert, C., DeRosse, P., Burdick, K. E., Morgan, T. V., Kane, J. M. et al. Runs of homozygosity reveal highly penetrant recessive loci in schizophrenia. Proc. Nat Acad. Sci. USA 104, 19942-19947 (2007).

16 Walsh, T., McClellan, J. M., McCarthy, S. E., Addington, A. M., Pierce, S. B., Cooper, G. $M$. et al. Rare structural variants disrupt multiple genes in neurodevelopmental pathways in schizophrenia. Science 320, 539-543 (2008).

17 Huang, D. W., Sherman, B. T. \& Lempicki, R. A. Systematic and integrative analysis of large gene lists using DAVID bioinformatics resources. Nat. Protoc. 4 , 44-57 (2008).

18 Dennis, G., Sherman, B., Hosack, D., Yang, J., Gao, W., Lane, H. C. et al. DAVID: database for annotation, visualization, and integrated discovery. Genome Biol. 4, P3 (2003).

19 Chen, Q- Y., Chen, Q., Feng, G- Y., Lindpaintner, K., Chen, Y., Sun, X. et al. Case-control association study of the close homologue of L1 (CHL1) gene and schizophrenia in the Chinese population. Schizophr. Res. 73, 269-274 (2005).

20 Sakurai, K., Migita, O., Toru, M. \& Arinami, A. An association between a missense polymorphism in the close homologue of $\mathrm{L} 1(\mathrm{CHL} 1, C A L L)$ gene and schizophrenia. Mol. Psychiatry 7, 412-415 (2002).

21 lijima, Y., Inada, T., Ohtsuki, T., Senoo, H., Nakatani, M. \& Arinami, T. Association between chromogranin b gene polymorphisms and schizophrenia in the japanese population. Biol. Psychiatry 56, 10-17 (2004).

22 Zhang, B., Tan, Z., Zhang, C., Shi, Y., Lin, Z., Gu, N. et al. Polymorphisms of chromogranin B gene associated with schizophrenia in Chinese Han population. Neurosci. Lett. 323, 229-233 (2002)

23 Bray, N. J. Gene expression in the etiology of schizophrenia. Schizophr. Bull. 34, 412 418 (2008)

24 Riley, B. P. \& McGuffin, P. Linkage and associated studies of schizophrenia. Am. J. Med. Genet. 97, 23-44 (2000).

25 Badner, J. A. \& Gershon, E. S. Meta-analysis of whole-genome linkage scans of bipolar disorder and schizophrenia. Mol. Psychiatry 7, 405-411 (2002).

26 McGuffin, P., Tandon, K. \& Corsico, A. Linkage and association studies of schizophrenia. Curr. Psychiatry Rep. 5, 121-127 (2003).

27 Sachidanandam, R., Weissman, D., Schmidt, S. C., Kakol, J. M., Stein, L. D., Marth, G. et al. A map of human genome sequence variation containing 1.42 million single nucleotide polymorphisms. Nature 409, 928-933 (2001).

28 Shi, J., Levinson, D. F., Duan, J., Sanders, A. R., Zheng, Y. \& Pe'er, I. et al. Common variants on chromosome $6 \mathrm{p} 22.1$ are associated with schizophrenia. Nature 460, 753-757 (2009)

29 Nakata, K., Lipska, B. K., Hyde, T. M., Ye, T., Newburn, E. N., Morita, Y. et al. DISC1 splice variants are upregulated in schizophrenia and associated with risk polymorphisms. Proc. Natl Acad. Sci. USA 106, 15873-15878 (2009).

30 Bertolino, A., Fazio, L., Caforio, G., Blasi, G., Rampino, A., Romano, R. et al. Functional variants of the dopamine receptor D2 gene modulate prefronto-striatal phenotypes in schizophrenia. Brain 132, 417-425 (2009).

31 Zhao, C., Xu, Z., Chen, J., Yu, Z., Tong, K. L., Lo, W. S. et al. Two isoforms of GABAA receptor [beta]2 subunit with different electrophysiological properties: differential expression and genotypical correlations in schizophrenia. Mol. Psychiatry 11, 1092-1105 (2006).

32 Zhao, C., Xu, Z., Wang, F., Chen, J., Ng, S.- K., Wong, P.- W. et al. Alternative-splicing in the exon-10 region of GABA(A) receptor beta(2) subunit gene: relationships between novel isoforms and psychotic disorders. PLOS ONE 4, e6977 (2009).

33 Tan, H- Y., Nicodemus, K. K., Chen, Q., Li, Z., Brooke, J. K., Honea, R. et al. Genetic variation in AKT1 is linked to dopamine-associated prefrontal cortical structure and function in humans. J. Clin. Invest. 118, 2200-2208 (2008).
34 Iwayama, Y., Hashimoto, K., Nakajima, M., Toyota, T., Yamada, K., Shimizu, E. et al. Analysis of correlation between serum $d$-serine levels and functional promoter polymorphisms of GRIN2A and GRIN2B genes. Neurosci. Lett. 394, 101-104 (2006)

35 Martucci, L., Wong, A. H. C., De Luca, V., Likhodi, O., Wong, G. W. H., King, N. et al. Nmethyl-D-aspartate receptor NR2B subunit gene GRIN2B in schizophrenia and bipolar disorder: polymorphisms and mRNA levels. Schizophr. Res. 84, 214-221 (2006).

36 Benedum, U. M., Lamouroux, A., Konecki, D. S., Rosa, P., Hille, A., Baeuerle, P. A. et al. The primary structure of human secretogranin I (chromogranin B): comparison with chromogranin A reveals homologous terminal domains and a large intervening variable region. EMBO J 6, 1203-1211 (1987).

37 Winkler, H. \& Fischer-Colbrie, R. The chromogranins A and B: the first 25 years and future perspectives. Neuroscience 49, 497-528 (1992).

38 Ozawa, H. \& Takata, K. The granin family-its role in sorting and secretory granule formation. Cell Struct. Funct. 20, 415-420 (1995).

39 Landén, M., Grenfeldt, B., Davidsson, P., Stridsberg, M., Regland, B. \& Gottfries, C.- G. et al. Reduction of chromogranin A and B but not $\mathrm{C}$ in the cerebrospinal fluid in subjects with schizophrenia. Eur. Neuropsychopharmacol. 9, 311-315 (1999).

40 Nowakowski, C., Kaufmann, W. A., Adlassnig, C., Maier, H., Salimi, K., Jellinger, K. A. et al. Reduction of chromogranin B-like immunoreactivity in distinct subregions of the hippocampus from individuals with schizophrenia. Schizophr. Res. 58, 43-53 (2002).

41 Giorgianni, F., Beranova-Giorgianni, S. \& Desiderio, D. M. Identification and characterization of phosphorylated proteins in the human pituitary. Proteomics 4, 587-598 (2004).

42 Blennow, K., Davidsson, P., Wallin, A. \& Ekman, R. Chromogranin A in cerebrospinal fluid: a biochemical marker for synaptic degeneration in alzheimer's disease? Dement. Geriatr. Cogn. Disord. 6, 306-311 (1995).

43 van Kammen, D. P., O'Connor, D. T., Neylan, T. C., Mouton, A., Gurklis, J. A. \& Gilbertson, M. W. et al. CSF chromogranin A-like immunoreactivity in schizophrenia: relationships with REM latency and slow wave sleep. Psychiatry Res. 42, 53-63 (1992)

44 van Kammen, D. P., Peters, J., Yao, J., Neylan, T., Beuger, M., Pontius, E. et al. CSF chromogranin A-like immunoreactivity in schizophrenia. Assessment of clinical and biochemical relationships. Schizophr. Res. 6, 31-39 (1991).

45 Miller, C., Kirchmair, R., Troger, J., Saria, A., Fleischhacker, W. W., Fischer-Colbrie, R. et al. CSF of neuroleptic-naive first-episode schizophrenic patients: Levels of biogenic amines, substance $P$, and peptides derived from chromogranin A (GE-25) and secretogranin II (secretoneurin). Biol. Psychiatry 39, 911-918 (1996).

46 Qi, Z., Miller, G. W. \& Voit, E. O. Computational analysis of determinants of dopamine (DA) dysfunction in DA nerve terminals. Synapse 63, 1133-1142 (2009).

47 Zucker, M., Valevski, A., Weizman, A. \& Rehavi, M. Increased platelet vesicular monoamine transporter density in adult schizophrenia patients. Eur. Neuropsychopharmacol. 12, 343-347 (2002).

48 Gutiérrez, B., Rosa, A., Papiol, S., Arrufat, F. J., Catalán, R., Salgado, P. et al. Identification of two risk haplotypes for schizophrenia and bipolar disorder in the synaptic vesicle monoamine transporter gene (SVMT). Am. J. Med. Genet. B Neuropsychiatr. Genet. 144B, 502-507 (2007).

49 Moskvina, V., Craddock, N., Holmans, P., Nikolov, I., Pahwa, J. S., Green, E. et al. Gene-wide analyses of genome-wide association data sets: evidence for multiple common risk alleles for schizophrenia and bipolar disorder and for overlap in genetic risk. Mol. Psychiatry 14, 252-260 (2008).

50 Wray, S. M., Stone, N. R., Visscher, J. L., O'Donovan, P. M., Sullivan, M. C. \& Sklar, P. F. $\mathrm{P}$ Common polygenic variation contributes to risk of schizophrenia and bipolar disorder. Nature 460, 748-752 (2009).

51 Liu, D., Chan, S., de Souza-Pinto, N., Slevin, J., Wersto, R., Zhan, M. et al. Mitochondrial UCP4 mediates an adaptive shift in energy metabolism and increases the resistance of neurons to metabolic and oxidative stress. Neuromolecular Med. 8, 389-413 (2006).

52 Rezin, G., Amboni, G., Zugno, A., Quevedo, J. \& Streck, E. Mitochondrial dysfunction and psychiatric disorders. Neurochemical Res. 34, 1021-1029 (2009).

53 Smorodchenko, A., Rupprecht, A., Sarilova, I., Ninnemann, O., Bräuer, A. U., Franke, $\mathrm{K}$. et al. Comparative analysis of uncoupling protein 4 distribution in various tissues under physiological conditions and during development. Biochim. Biophys. Acta 1788, 2309-2319 (2009).

54 Chan, S. L., Liu, D., Kyriazis, G. A., Bagsiyao, P., Ouyang, X. \& Mattson, M. P. Mitochondrial uncoupling protein-4 regulates calcium homeostasis and sensitivity to store depletion-induced apoptosis in neural cells. J. Biol. Chem. 281, 37391-37403 (2006)

55 Harms, N., Ras, J., Reijnders, W., van Spanning, R. \& Stouthamer, A. S-formylglutathione hydrolase of Paracoccus denitrificans is homologous to human esterase $D$ : a universal pathway for formaldehyde detoxification? J. Bacteriol. 178, 6296-6299 (1996).

56 Abuaf, N. Neurological syndromes associated with nervous system-specific autoantibodies. Clin. Rev. Allergy Immunol. 19, 1-5 (2000).

57 Young, L.- J., Lee, E. Y., To, H. A., Bookstein, R., Shew, J.- Y., Donoso, L. A. et al. Human esterase D gene: complete cDNA sequence, genomic structure, and application in the genetic diagnosis of human retinoblastoma. Human Genet. 79, 137-141 (1988).

58 Srinivas, K., Sinha, S., Taly, A. B., Prashanth, L. K., Arunodaya, G. R., Janardhana Reddy, Y. C. et al. Dominant psychiatric manifestations in Wilson's disease: A diagnostic and therapeutic challenge. J. Neurol. Sci. 266, 104-108 (2008). 
59 Gasque, P. Complement: a unique innate immune sensor for danger signals. Mol. Immunol. 41, 1089-1098 (2004).

60 Isenman, D. E. \& Young, J. R. The molecular basis for the difference in immune hemolysis activity of the Chido and Rodgers isotypes of human complement component C4. J Immunol 132, 3019-3027 (1984).

61 Law, S. K., Dodds, A. W. \& Porter, R. R. A comparison of the properties of two classes, C4A and C4B, of the human complement component C4. EMBO J 3, 1819-1823 (1984).

62 Carroll, M. C., Belt, T., Palsdottir, A. \& Porter, R. R. Structure and organization of the C4 genes. Philos. Trans. R. Soc. Lond. B Biol. Sci. 306, 379-388 (1984).

63 Rudduck, C., Beckman, L., Franzen, G., Jacobsson, L. \& Lindstrom, L. Complement factor C4 in schizophrenia. Hum. Hered. 35, 223-226 (1985).

64 Mayilyan, K. R., Dodds, A. W., Boyajyan, A. S., Soghoyan, A. F. \& Sim, R. B. Complement C4B protein in schizophrenia. World J. Biol. Psychiatry 9, 225-230 (2008).

65 Schroers, R., Nothen, M. M., Rietschel, M., Albus, M., Maier, W., Schwab, S. et al. Investigation of complement C4B deficiency in schizophrenia. Hum. Hered. 47, 279-282 (1997).

66 Ursic, D., Sedbrook, J., Himmel, K. \& Culbertson, M. The essential yeast Tcp1 protein affects actin and microtubules. Mol. Biol. Cell 5, 1065-1080 (1994).

67 Glantz, L. A. \& Lewis, D. A. Decreased dendritic spine density on prefrontal cortical pyramidal neurons in schizophrenia. Arch. Gen. Psychiatry 57, 65-73 (2000).

68 Schlatter, M. C., Buhusi, M., Wright, A. G. \& Maness, P. F. CHL1 promotes Sema3Ainduced growth cone collapse and neurite elaboration through a motif required for recruitment of ERM proteins to the plasma membrane. J. Neurochem. 104, 731-744 (2008).

69 Chen, S., Mantei, N., Dong, L. \& Schachner, M. Prevention of neuronal cell death by neural adhesion molecules L1 and CHL1. J. Neurobiol. 38, 428-439 (1999).

70 Angeloni, D., Lindor, N. M., Pack, S., Latif, F., Wei, M.- H. \& Lerman, M. I. CALL gene is haploinsufficient in a 3p- syndrome patient. Am. J. Med. Genet. 86, 482-485 (1999).

71 Shuib, S., McMullan, D., Rattenberry, E., Barber, R. M., Rahman, F., Zatyka, M. et al. Microarray based analysis of 3p25-p26 deletions (3p-syndrome). Am. J. Med. Genet. A 149A, 2099-2105 (2009).

72 Montag-Sallaz, M., Baarke, A. \& Montag, D. Aberrant neuronal connectivity in CHL1deficient mice is associated with altered information processing-related immediate early gene expression. J. Neurobiol. 57, 67-80 (2003).

$73 \mathrm{Li}, \mathrm{L} ., \mathrm{Du}, \mathrm{Y}$., Li, N., Wu, X. \& Wu, Y. Top-down modulation of prepulse inhibition of the startle reflex in humans and rats. Neurosci. Biobehav. Rev. 33, 1157-1167 (2009).

74 Uchida, N., Shimamura, K., Miyatani, S., Copeland, N. G., Gilbert, D. J., Jenkins, N. A. et al. Mouse (alpha)N-catenin: two isoforms, specific expression in the nervous system, and chromosomal localization of the gene. Dev. Biol. 163, 75-85 (1994).

75 Park, C., Falls, W., Finger, J. H., Longo-Guess, C. M. \& Ackerman, S. L. Deletion in Catna2, encoding alpha $\mathrm{N}$-catenin, causes cerebellar and hippocampal lamination defects and impaired startle modulation. Nat. Genet. 31, 279-284 (2002).

76 Abe, K., Chisaka, O., Van Roy, F. \& Takeichi, M. Stability of dendritic spines and synaptic contacts is controlled by alpha N-catenin. Nat. Neurosci. 7, 357-363 (2004).

Supplementary Information accompanies the paper on Journal of Human Genetics website (http://www.nature.com/jhg) 